\title{
Recent Advances in Mobile Middleware for Wireless Systems and Services
}

\author{
Paolo Bellavista • Jiang (Linda) Xie • Tuna Tugcu
}

Published online: 8 November 2008

(C) Springer Science + Business Media, LLC 2008

It is not debatable that mobile services, i.e., service provisioning not only to mobile portable devices with possibly multiple heterogeneous wireless interfaces but also depending on different aspects of mobile users' contexts (client location, local resource availability, portable device characteristics, ...), are expected to be a tremendously relevant business market in the very next years. The recent progresses in wireless technologies and computing miniaturization have permitted to enable today's pervasive and ubiquitous computing scenarios that were considered science-fiction only a few years ago. But that is a relatively old story...

The novel aspect of the last couple of years is that it starts to be widely recognized that the next crucial technological challenge in mobile services is to identify, propose, validate, and spread the adoption of open and interoperable software-support solutions specifically designed and implemented for wireless-enabled portable devices. Only such support solutions, called mobile middlewares in the following, can allow to accelerate and

P. Bellavista $(\bowtie)$

Dept. Electronics Computer Science and Systems (DEIS), University of Bologna,

Viale Risorgimento, 2,

40136 Bologna, Italy

e-mail: paolo.bellavista@unibo.it

URL: http://ia.deis.unibo.it/Staff/PaoloBellavista/

J. Xie

University of North Carolina,

Charlotte, NC, USA

e-mail: linda.xie@uncc.edu

T. Tugcu

Bogazici University,

Istanbul, Turkey

e-mail: tugcu@boun.edu.tr simplify the development and deployment of mobile services over heterogeneous devices, by leaving service developers ideally with the only burden of concentrating their efforts on application logic, without taking into account the implementation issues related to collecting and processing context data over heterogeneous environments, properly and synergically managing several heterogeneous wireless interfaces and the deriving multi-homing opportunities, integrating and orchestrating heterogeneous service components possibly discovered at runtime, and so on. Only the availability of novel mobile middlewares can allow to effectively support these functions with minimum impact on mobile service realization, thus enabling the reduction of development costs and accelerating time-tomarket. In addition, the spreading of a given middleware platform within the community of mobile service developers is going to represent one of the most crucial strategic advantages for any of the players (device manufacturers, service developers, service providers, and of course middleware developers) involved in the rich field of mobile services. That is manifestly confirmed by the relevant group of industrial players that, more or less recently, have decided to enter the battlefield of mobile middlewares, from the general-purpose early work of Sun's Java Micro Edition and Microsoft Windows Mobile, to the recent Google Android project and the Apple iPhone Software Development Kit.

With this perspective in mind, in 2008 we decided to launch a new conference, International Conference on MOBILe Wireless MiddleWARE, Operating Systems, and Applications (Mobilware). The response from the academic/ industrial communities was excellent, with a large number of high-quality technical submissions notwithstanding the first year of the event. The technical program of Mobilware 2008 well reflects the current priorities in mobile middlewares: several papers address network, systems, and service 
management issues in dependence of location and context; several contributions relate to support infrastructure properties, such as middleware architectures and security; a considerable part of the program is devoted to consolidated and emerging applications for mobile services, such as multimedia, wireless sensor and vehicular network applications, and surveillance systems; finally, lower-layer issues such as autonomic interface management, advanced forms of vertical handoffs, mobility modeling, and applicationspecific communication encoding have also attracted the attention of conference authors. Given the high quality of the papers accepted for presentation at Mobilware 2008 and thanks to the encouraging comments received by the MONET Editor-in-Chief, we decided to organize this special issue with extended versions of the papers selected from the best submissions to Mobilware 2008, with the final goal of giving a fresh and up-to-date overview of several primary research directions in the field.

This issue starts with two original contributions that address two crucial communication-related issues for the realization of effective middlewares for wireless portable devices. The first article entitled "Middleware for MultiInterfaces Management Through Profiles Handling" investigates novel profile-based solutions for guiding the management of different heterogeneous interfaces (WiFi, Bluetooth, UMTS, ...), which are usually available in nowadays mobile terminals. The second paper, which is entitled "Mobility-aware Management of Internet Connectivity in Always Best Served Wireless Scenarios", instead focuses on the definition of novel, mobility-aware, and scarcely intrusive monitoring indicators to effectively take decisions on which Internet connectivity opportunity to exploit at runtime.

After that part devoted to how mobile middleware solutions can exploit low-layer communication visibility to enable the most suitable management strategies, the special issue devotes a relevant part of its space to describe state-of-the-art solutions about middleware organization, architecture, and implementation. In particular, "MobiSoC: A Middleware for Mobile Social Computing Applications" describes a notable experience in designing and implementing a middleware platform for the support of mobile services with the focus on one specific application class (social networking applications), while "Recoverable Class Loaders for a Fast Restart of Java Applications" addresses the specific implementation issue of improving the performance of Java applications through the proper design of novel class loaders for portable devices. Non-functional properties, such as the support of flexible and extensible security, are increasingly relevant to determine the commercial success and widespread adoption of mobile middleware solutions: "Privacy from Promises to Protection-Privacy Guaranteeing Execution Container" addresses the specific issue of achieving privacy through proper container-based architectural choices.

The last part of the special issue includes three papers that give a variegated overview of some of the possible application domains where mobile services could relevantly benefit from the mobile middleware support. " $A n$ Activity Recognition System for Mobile Phones" describes how to exploit an off-the-shelf mobile phone and a few accelerometers to implement a simple middleware-based mobile service that recognizes the activities a user is performing; "Monitoring Smartphones for Anomaly Detection" employs mobile phones to promptly detect anomaly situations that differ from a defined regular behavior; "Extracting High-Level Information from Location Data: the W4 Diary Example" describes how it is possible to simply infer high-level context data based on profile and location information, thus providing mobile services with easy-to-use context at the proper level of abstraction.

Finally, we want to express our gratitude to all the contributors and to all the reviewers whose accurate work was crucial for the realization of this special issue. An enormous and special thanks goes to Professor Imrich Chlamtac who trusted in the Mobilware conference series from the beginning and gave us invaluable support and suggestions. Thanks to all of them, we believe that this collection of selected papers as a whole can pleasantly introduce the readers to the challenging arena of mobile middlewares and can help in giving a fresh sketch of currently hot topics and state-of-the-art solutions in the field.

Enjoy your reading!

Paolo Bellavista (paolo.bellavista@unibo.it) is an Associate Professor of computer engineering at the University of Bologna, where he has received his Laurea degree and $\mathrm{PhD}$. He is senior member of the IEEE, member of the ACM and the ICST, and serves in the Editorial Boards of the IEEE Communications Magazine, the IEEE Transactions of Services Computing, the Springer Journal of Network and Systems Management, and the Elsevier Journal of System Architecture. He has chaired several international conferences, including IEEE ISCC'06, ICST Mobilware'08, and different tracks in IEEE COMPSAC ('07 and '08). His research activities span from mobile computing to mobile agent-based middlewares, from pervasive wireless computing to location/context-aware services, from vehicular/sensor wireless ad hoc networks to adaptive multimedia. He is co-editor of the "Handbook of Mobile Middleware" and authored more than 110 journal/conference papers. Additional information at: http://lia.deis.unibo.it/Staff/Paolo Bellavista/

Jiang (Linda) Xie (linda.xie@uncc.edu) is an Assistant Professor of Electrical and Computer Engineering at the University of North Carolina-Charlotte. She received her B.E. degree from Tsinghua University, M.Phil degree from Hong Kong University of Science and 
Technology, and M.S. and Ph.D. degrees from Georgia Institute of Technology, all in electrical engineering. She is a member of IEEE and ACM, and serves on the Editorial Board of Elsevier Computer Networks Journal and Elsevier Journal of Network and Computer Applications. She has served and is serving in the organizing committees of several international conferences, including IEEE GLOBECOM 2009, ICST Mobilware 2008, ICST WICON 2008, ICST ChinaCom 2008, IEEE ICCCN 2008 and 2007. Her research interests include mobility management, resource management, QoS management of wireless networks and the next generation Internet. Additional information: http://www.coe.uncc.edu/ jxie1/
Tuna Tugcu (tugcu@boun.edu.tr) is an Assistant Professor of computer engineering at the Bogazici University, where he has received his BS and $\mathrm{PhD}$ degrees. He worked as a post-doc, and then as a visiting professor at Georgia Institute of Technology. He is member of the IEEE, member of the ACM, and serves in the Editorial Board of the Elsevier Computer Networks Journal. He has served in the organizational committees of several international conferences, and co-chaired ICST Mobilware'08. His research interests include wireless networks, with the emphasis on Next Generation Wireless Networks including WiMAX, MBWA, cognitive radio, and sensor networks. Additional information at: http://www.cmpe.boun.edu.tr/ tugcu/ 